\title{
Site-specific strategies stylize streptavidin
}

These new, The high-affinity interaction engineered specific binding protein-either Streptomyces avidinii streptstreptavidins $\begin{aligned} & \text { avidin or egg white avidin-has } \\ & \text { found wide application in bio- }\end{aligned}$ technology. Native streptavidin is a tetrameric molecule with four equivalent binding sites for biotin. Protein engineering approaches, as reported in"Engineered Chimeric Streptavidin Tetramers as Novel Tools for Bioseparations and Drug Delivery," by Chilkoti et al. on p. 1198 of this issue of Bio/Technology, have now produced site-specifically modified molecules with altered ligand binding affinity and/or nonequivalent binding sites within a single tetrameric molecule. These modified molecules have the potential for greatly expanded utility in biotechnology.

Natural streptavidin and avidin are very stable molecules that are unusually resistant to oxidative, proteolytic, or thermal degradation. Both proteins reversibly bind the water soluble vitamin, biotin, with such affinity that the avidin:biotin interaction was once thought to be covalent. The tetrameric structure of these biotinbinding proteins is ideally suited for capture and labeling techniques that depend on multiple attachment sites. Equally importantly, the biotin ligand is readily derivatized under mild conditions; this allows it to be attached to a great variety of biological molecules, dyes, and solid substrates with relatively little effect on its binding affinity for streptavidin.

However, in one respect at least, the streptavidin-biotin interaction is not ideal. The high affinity association between the moleculesideal for capture and labelling limits the application of the system to nearly irreversible interactions. A streptavidin molecule that retained the high-affinity binding site for biotin, but that also containing a lower affinity site would facilitate more controlled binding of biotinylated ligands and thereby vastly increase the system's application. That is just what Patrick Stayton and his colleagues have developed. They prepared streptavidin tetramers containing both wild type and modified subunits by refolding from mixtures of protein-engineered and wild type polypeptides. The chimeric tetramers retain most positive attributes of the natural molecule.

Three modified streptavidin subunits have been prepared. One that contains a single, free cysteine allows any streptavidin tetramer that contains it to be conjugated to thiolcontaining reagents-such as preparations of targeting antibodies, therapeutics, or imaging agents. The second engineered subunit has a tryptophan-to-alanine substitution at the biotin-binding site: Chimeric tetramers containing it, therefore, have both high- and low-affinity biotin-binding sites and allow milder conditions to be used in capture and release applications. A third modification has been made to allow the assembly of modified or wild-type subunit in a controlled fashion. A histidine residue at the tetramer subunit interface was changed to cysteine. This allows the formation of an intersubunit disulfide bond and confers the possibility of accurately controlling the relative orientation and stoichiometry of subunits in the mixed molecule tetramers.

The intrinsic properties of both protein and ligand have made the native streptavidin/biotin system a practical tool for biotechnology. These new, engineered streptavidins represent a significant step toward its expanded use in new applications that may exploit a new level of binding diversity and selection.

Patricia Weber is senior director of structural chemistry, ScheringPlough Research Institute, 2015 Galloping Hill Road, Kenilworth, NJ 07033.

\section{Mammalian cell technology: Secretion on demand}

\begin{abstract}
Producing authentic, homogeous proteins in heterologous cells has never been quite as straightforward as the phrase "recombinant gene expression" might suggest. In "Regulated Secretion of Prolactin by the Mouse Insulinoma Cell Line $\beta$ TC-3," beginning on p.1191 in this issue of Bio/Technology, Chen et al. have put a new wrinkle on an old prune by demonstrating that the host cells' natural processing mechanisms can be mobilized to the cause.
\end{abstract}

Literature searches confined to the past ten years would fail to pick up the pioneering efforts of biotechnologists who overcame many of the problems of polypeptide secretion from the yeast Saccharomyces cerevisiae, the early eukaryotic workhorse of the biotechnology industry. Indeed, each of the big three Californian biotechnology companies-Amgen (Thousand Oaks), Genentech (S. San Francisco) and Chiron (Emeryville)-developed systems that utilized the efficiency of the endogenous Saccharomyces KEX2 subtilisin-like protease for authentic processing of peptide hormone precursor proteins at either natural or engineered dibasic processing sites. Thus, the heterogeneity of cleavage by signal peptidase and the more esoteric STE13 gene product was counteracted in one fell swoop. As the need for "authentic" glycosylation patterns on potential therapeutic proteins emerged, and the emphasis shifted 